\title{
The dynamics of memory: Context-dependent updating
}

\author{
Almut Hupbach, ${ }^{1,3}$ Oliver Hardt, ${ }^{2}$ Rebecca Gomez, ${ }^{1}$ and Lynn Nadel ${ }^{1}$ \\ ${ }^{1}$ Department of Psychology, The University of Arizona, Tucson, Arizona 85721, USA; ${ }^{2}$ Department of Psychology, \\ McGill University, Montreal, Quebec H3A 2T5, Canada
}

\begin{abstract}
Understanding the dynamics of memory change is one of the current challenges facing cognitive neuroscience. Recent animal work on memory reconsolidation shows that memories can be altered long after acquisition. When reactivated, memories can be modified and require a restabilization (reconsolidation) process. We recently extended this finding to human episodic memory by showing that memory reactivation mediates the incorporation of new information into existing memory. Here we show that the spatial context plays a unique role for this type of memory updating: Being in the same spatial context during original and new learning is both necessary and sufficient for the incorporation of new information into existing episodic memories. Memories are automatically reactivated when subjects return to an original learning context, where updating by incorporating new contents can occur. However, when in a novel context, updating of existing memories does not occur, and a new episodic memory is created instead.
\end{abstract}

Memory does not provide a perfect record of the past and can be altered long after acquisition. This malleability of memory has important implications, for public and private spheres of life. The dynamic nature of memory is probably not a design flaw; it can allow us to update existing knowledge in light of new information. Understanding the dynamics of memory change is one of the current challenges facing cognitive neuroscience, but until recently we have lacked experimental approaches to address this problem, and the neural mechanisms controlling memory updating remain obscure. Recent work on the phenomenon of memory "reconsolidation" provides a methodological approach to disentangling the processes involved in this form of memory modification (Dudai 2006).

It has long been assumed that initially labile memory traces are transformed into relatively permanent ones by a timedependent stabilization process called memory consolidation (McGaugh 2000). The permanence of consolidated traces has been called into question by studies showing that memories can become labile for some time after reactivation (e.g., Misanin et al. 1968; Nader et al. 2000). It is assumed that memories in this state need to be reconsolidated or they will be lost. It is possible that in this transient plastic state, memories are also open to modification. Consistent with this view, reconsolidation effects have been demonstrated in a variety of animal protocols and also in human procedural memory (Walker et al. 2003).

On the assumption that reconsolidation underlies the malleability of memory, we recently asked whether reactivation is involved in the updating of human episodic memory (Hupbach et al. 2007). Subjects learned a set of objects in session 1. Fortyeight hours later (session 2), subjects were either reminded of the first session or not and immediately afterward learned a second set of objects. Again 48 h later (session 3), subjects were asked to recall the first set only, that is, the objects learned in session 1 . Reminded subjects showed a high number of intrusions from set 2 when recalling set 1 in session 3 , while subjects who had not been reminded showed almost no intrusions, demonstrating that the updating of pre-existing memory is dependent on reactivation of that memory. Importantly, this effect takes time to

\footnotetext{
${ }^{3}$ Corresponding author.
}

E-mail ahupbach@email.arizona.edu; fax (520) 621-9306.

Article is online at http://www.learnmem.org/cgi/doi/10.1101/lm.1022308. emerge (i.e., it was not evident immediately after learning set 2 in session 2) and is unique to the reactivated list (no intrusions from set 1 were observed when recalling set 2 ). In other words, retroactive interference and source memory accounts cannot readily explain these findings: the former because such interference is seen sooner rather than later, the exact opposite of the pattern we observed, and the latter because source confusion should lead to intrusions in both directions (set 1 into set 2 and vice versa), which we fail to see.

While these findings provide the first demonstration of reconsolidation in episodic memory, this study did not address precisely what factors led to memory reactivation and thus were critical in mediating plasticity and updating of episodic memory. The stimulus qualities required for memory reactivation bear directly on our understanding of the organization of memory and the nature of memory dynamics. In our initial study described above, the reminder comprised three different components: spatial context, experimenter, and reminder question. Subjects in the reminder group were brought back to the same spatial context, where they met the same experimenter, and were asked a reminder question before learning the second set. For the subjects that were not reminded, all three components were omitted. We now asked which of these components are critical for reactivating the memory representation, allowing for updating of human episodic memory. On most theoretical accounts, including encoding-specificity (Tulving and Thomson 1973) and transferappropriate processing (Morris et al. 1977), any (or all) of these components could serve as cues for accessing or reactivating the memory trace of the first learning experience. We hypothesized, however, that spatial context might play a particularly important role. We have argued that spatial context is not a memory cue in the standard associative sense (Nadel and Willner 1980). Instead, context serves as a "scaffold" for a configural representation of all the elements in an experienced event-that is, an episodic memory. When returned to a particular context, this memory is reactivated, allowing it to be updated as a function of new experiences in that context. This could produce intrusions from the second set into the first one.

In a series of three experiments we explored the contribution of the different reminder components (Experiment 1), their combination (Experiment 2), and variations of the spatial context reminder (Experiment 3). Despite the different reminders 
that were implemented in session 2, all other aspects of the experimental procedure were identical to our previous study. In a fourth experiment, we explored the role of the retrieval context in session 3: Specifically, we asked whether the reconsolidated memory is context dependent.

\section{Experiment 1}

The first experiment looked separately at the three components of the reminder that we used in our previous study (experimenter, spatial context, and reminder question).

\section{Method}

\section{Design and participants}

The only independent variable that was varied between subjects was the reminder in session 2: The question group met a different experimenter in a different room than in session 1 but was asked to describe the set-learning procedure experienced in that session. The experimenter group met the same experimenter in a different room and was not asked a reminder question. The context group returned to the same room but met a different experimenter and was not asked a reminder question.

A total of 36 undergraduate students from the University of Arizona participated in the experiment. They received course credit for participation. Twelve subjects were randomly assigned to each condition. All experimental procedures were approved by the Internal Review Board of the University of Arizona.

\section{Materials}

Set 1 and set 2 each consisted of 20 unrelated objects (see Appendix).

\section{Procedure}

All subjects learned a set of 20 unrelated objects during session 1 . The objects were sorted in front of the subject into a distinctive blue basket. The subjects' task was to name each object. After the presentation, subjects were asked to recall as many objects as possible. If they recalled less than 17 objects, the objects were presented again. This procedure continued until subjects could recall at least 17 objects or until a maximum of four learning trials was reached. Forty-eight hours later (session 2), equal numbers of subjects were randomly assigned to an experimental condition. The question group met a different experimenter in a room different from the one used session 1. The blue basket was shown, and subjects were asked the following questions: "Do you remember this basket? Can you describe the general procedure of what you did on Monday?" (As intended, subjects did not spontaneously recall specific items from set 1 ). The experimenter group met the same experimenter in a different room and was not asked a reminder question. The context group returned to the same room but met a different experimenter and was not asked a reminder question. All subjects in session 2 then learned a second set of 20 unrelated objects. The procedure differed from that during session 1 so the task would not serve as a reminder. All objects were placed in front of the subjects, who were asked to name each of the objects and were given 30 additional seconds to study them. Then the objects were removed, and subjects were tested for immediate recall. The same learning criterion as during session 1 was used (at least 17 objects or a maximum of four learning trials). Finally, $48 \mathrm{~h}$ later (session 3 ), all subjects returned to the room in which they had learned set 1 and met the same experimenter as during session 1 . They were asked to recall as many objects as possible from the first set. This procedure was repeated for a total of four consecutive recall trials.

\section{Results}

In this and the following experiments, the mean percentage of objects recalled from set 1 (averaged over all four recall trials) and the mean percentage of items falsely intermixed from set 2 (intrusions, averaged over all four recall trials) served as dependent variables. Results of the experiments are displayed in Figure 1. Recall performance was similar in all three groups $(F<1)$ (Fig.
A
Experiment 1
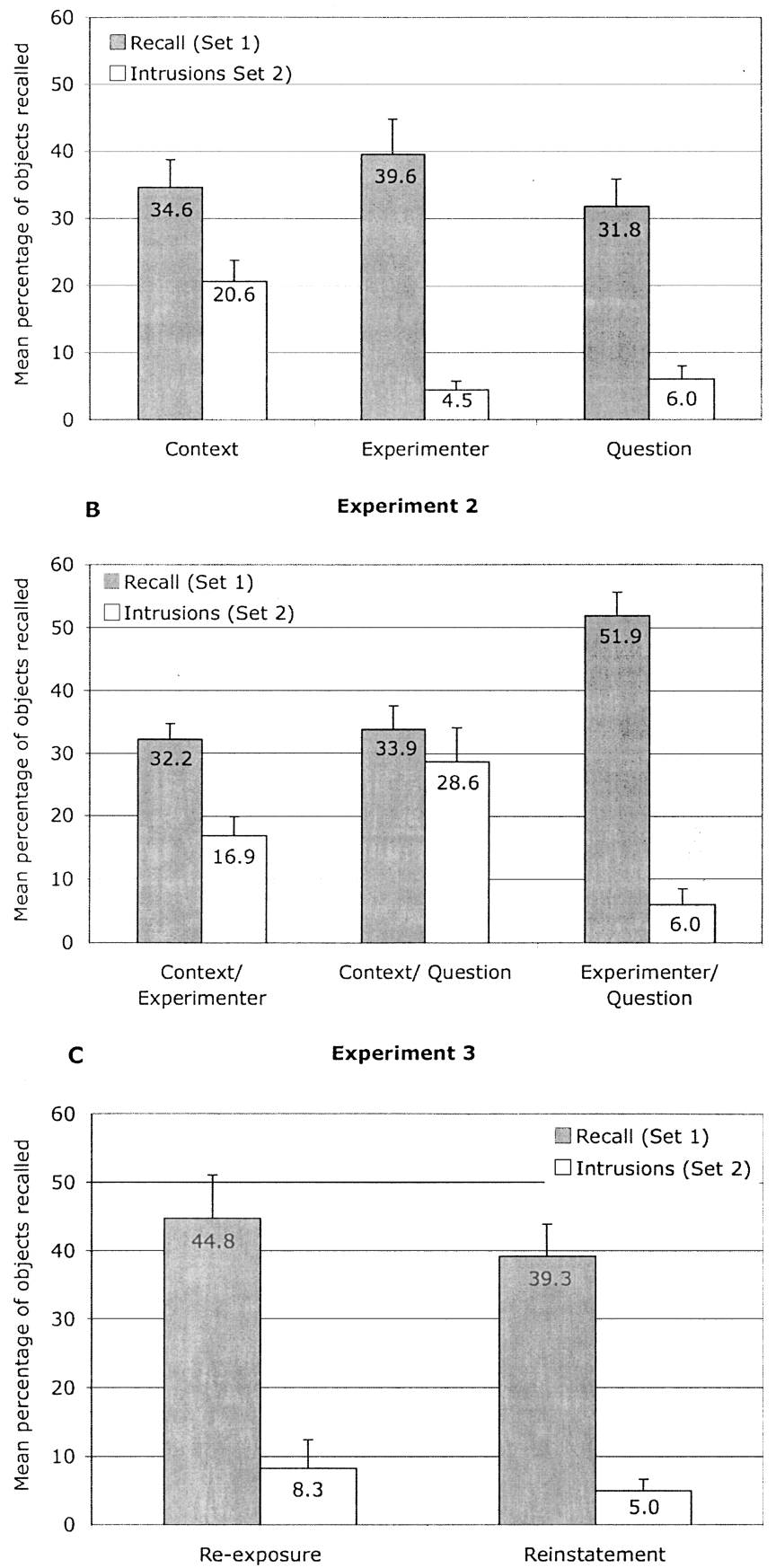

Figure 1. Mean percentage of objects correctly and falsely recalled in session 3 in Experiments 1-3 (Fig. 1A,B,C, respectively). Error bars, SEM. Note that subjects were asked to recall objects from set 1 . Objects that were falsely recalled from set 2 are labeled as intrusions. 
1A), but the number of intrusions from set 2 into set 1 differed between the groups $\left(F_{(2,33)}=16.00, M S E=59.45, P<0.01\right)$ (Fig. 1A). The context group showed higher levels of intrusions than both the experimenter and the question groups $(P<0.05$, for both comparisons, Dunnett's $C)$, which did not differ from each other $(P>0.05)$.

\section{Discussion}

Intrusions were only observed in the group that learned set 2 in the same spatial context in which they had learned set 1 . Neither meeting the same experimenter nor being asked a reminder question triggered memory updating. However, it is possible that spatial context is simply a stronger reminder than the other two components, which might need to be combined in order to cause intrusions. In a second experiment, we therefore tested all possible combinations of two reminder components.

\section{Experiment 2}

\section{Method}

The method was identical to the one used in Experiment 1 with the exception that instead of individual reminder components, all possible combinations of two reminder components were administered during session 2: For the experimenter/question group, the same experimenter as in session 1 asked the reminder question but in a different room than before. The context/experimenter group met the same experimenter in the same room as in session 1 , but the reminder question was omitted. The context/question group returned to the same room and was asked the reminder question, but a different experimenter administered the procedure.

A total of 36 undergraduate students from the University of Arizona participated in the experiment. They received course credit for participation. Twelve subjects were randomly assigned to each condition.

\section{Results}

The groups differed in their recall performance $\left(F_{(2,33)}=10.87\right.$; $M S E=131.64, P<0.01$ ) (Fig. 1B) such that the experimenter/ question group recalled more items than the context/question and context/experimenter groups (Tukey-HSD, $P<0.01$, for both comparisons). Importantly, the number of intrusions (Fig. 1B) differed between the groups $\left(F_{(2,33)}=7.75 ; M S E=197.99, P<0.01\right)$. Post-hoc tests (Dunnett's C) showed that the context/experimenter and context/question group did not differ in the number of intrusions $(P>0.05)$, but both groups showed significantly higher levels of intrusions than the experimenter/question group $(P<0.05$, for both comparisons).

\section{Discussion}

The experimenter/question group recalled more items than the context/question and context/experimenter groups. However, because the experimenter/question group also recalled more objects from set 1 than the reminder group that received a threecomponent reminder (question/experimenter/context) in our previous study (Hupbach et al. 2007), the result appears to reflect random fluctuation in recall performance.

The memory-updating effect (intrusions from set 2 into set 1) was found in both groups experiencing the same context, but the magnitude of the effect did not exceed that of the contextonly group in Experiment 1 . Thus, spatial context appears to be a necessary and sufficient reminder causing incorporation of new information into existing memory.

\section{Experiment 3}

Recent work shows that the hippocampus, critical for representing spatial context (O'Keefe and Nadel 1978; Nadel and Willner 1980), is also involved in imagining events (Hassabis et al. 2007). When asked to dream up certain experiences, such as lying at the beach, the verbal reports of amnesic patients with bilateral hippocampal damage differ significantly from the descriptions provided by healthy individuals. Unlike the healthy controls, the imagined events of amnesic patients lack spatial resolution and detail. Because of the role of the hippocampus in both spatial memory and spatial imagination, we explored in a third experiment to determine whether mentally reinstating the spatial context in which the original set was learned is sufficient to induce plasticity or whether one must actually be present in the room to observe this effect. We addressed this issue in two different ways. In one condition subjects mentally reinstated the spatial context in which they had learned set 1 by recalling specific features of the room and drawing a map of the room in which set 1 had been learned while in a different room. In a second condition, subjects were briefly reexposed to the original test room but were then brought to a different room where they learned the second set.

\section{Method}

The method was identical to the one used in Experiments 1 and 2 with the exception of the reminder conditions realized in session 2. Subjects were randomly assigned to one of two different conditions. For the reinstatement group, subjects were seated in a different room in session 2 , and before learning set 2, they were asked to draw a map and to describe the room in which they had learned set 1 . The reexposure group briefly revisited the original test room (2-3 $\mathrm{min}$ ) and was then brought to a different room in which they learned set 2 . In both groups, subjects met a different experimenter and were not asked a reminder question.

A total of 24 undergraduate students from the University of Arizona participated in the experiment. They received course credit for participation. Twelve subjects were randomly assigned to each condition.

\section{Results}

A comparison between the reinstatement, the reexposure group, and the context group of Experiment 1 (Fig. 1C) showed no group differences in recall performance $\left(F_{(2,33)}=1.25 ; M S E=262.00\right.$, $P=0.30)$ but showed significant differences in the number of intrusions $\left(F_{(2,33)}=12.55 ; M S E=74.81, P<0.01\right)$. Both the reinstatement and the reexposure groups showed significantly fewer intrusions than the context group (Tukey-HSD, $P<0.01$ for both comparisons), and their intrusion rates did not differ from each other $(P=0.98)$.

\section{Discussion}

Experiment 3 shows that the updating effect does not occur when subjects briefly revisit the test room in which they had encoded set 1 before learning set 2 in a different room. Similarly, imagining the old encoding context before learning set 2 does not cause intrusions. Together with results of Experiments 1 and 2, Experiment 3 suggests that subjects have to learn set 2 in the same spatial context in which set 1 was encoded in order for set 2 objects to be incorporated into set 1 memory. Interestingly, this result contrasts with a related finding in the area of contextdependent memories: Here, mental reinstatement of the test context in which the initial learning took place diminishes or eliminates the context dependence of memories (Smith and Vela 2001).

In second-order fear conditioning in rats, Debiec et al. (2006) showed that only directly reactivated memories undergo 
reconsolidation, whereas an indirect reactivation through the associative chain does not induce plasticity. Similarly, mental reinstatement could be viewed as a more indirect reactivation that is not as powerful as directly reexperiencing the spatial context during the learning of the second set. Why mental reinstatement is insufficient for the reactivation and updating of memories remains to be addressed in future studies.

\section{Experiment 4}

Experiments 1-3 suggest that the spatial context is crucial for the reactivation and subsequent updating and reconsolidation of memories. But our results are open to an alternative explanation, given that the relations between training, updating, and retrieval contexts varied between our critical groups. That is, our "context reminder" group, which showed an updating effect, was tested for retrieval of set 1 objects in the context of both original training of set 1 and subsequent training of set 2 . Our control group, which did not show an updating effect, was tested for retrieval of set 1 objects in a context different than that used during training of set 2. Perhaps these variations in the relation between set 2 training context (session 2), and retrieval context (session 3) played a critical role in our results. That is, perhaps subjects did not include set 2 items into their recall of set 1 because set 2 objects were not associated with the retrieval context.

The study of context-dependent memory has a long tradition in memory research. A meta analysis indicates that memory performance benefits from a stability between encoding and test context unless attention is drawn away from the test environment, for instance, by asking subjects to focus on other aspects of the to-be-remembered material such as inter-item associations (Smith and Vela 2001). Based on this result, we should expect better memory for set 1 when recall is performed in the same test environment in comparison to a different one. With regard to intrusions from set 2 into set 1, the predictions are less straightforward. On one hand, the intrusion effect found in our previous experiments could reflect the use of spatial context as a retrieval cue. Because the spatial context is associated with both set 1 and set 2 , set 2 items intrude into set 1 recall. ${ }^{4}$ In this case, a change of the test environment might encourage subjects to rely on different retrieval cues than the spatial context and, hence, might cause a decrease in intrusions. On the other hand, the reconsolidation account assumes that the reconsolidation process restabilizes the reactivated or updated memory, and while changing the test context could affect the ability to access this updated memory, it should not alter the updating effect itself.

Experiment 4 tested the effect of the test context by asking subjects to learn sets 1 and 2 in the same context (sessions 1 and 2 ) but to recall set 1 in a different test context during session 3. During session 2, half of the subjects received a reminder that consisted of all three components (spatial context, experimenter, reminder question), and half were reminded by the spatial context only.

\section{Method}

The method was similar to the one used in Experiment 1 with two exceptions: (1) During session 2, half of the subjects were brought back to the same spatial context, where they met the same experimenter, and were asked a reminder question before learning the second set (three-component reminder). The other half of the subjects returned to the same room but met a different experimenter and were not asked a reminder question (context reminder). (2) All subjects were brought to a different spatial con-

${ }^{4}$ Note, however, that this effect is unidirectional. In a previous study (Hupbach et al. 2007), we did not find intrusions from set 1 into set 2 when subjects were asked to recall set 2 . text (a different room) in session 3, where they were asked to recall set 1 objects only.

A total of 30 undergraduate students from the University of Arizona participated in the experiment. They received course credit for participation. Fifteen subjects were randomly assigned to each condition. We used a slightly higher number of subjects because of the emergence of a bimodal distribution in the number of intrusions (see Results section).

\section{Results}

The mean number of objects recalled from set 1 and the mean number of intrusions falsely recalled from set 2 did not differ in the context and the three-component reminder group $(t<1$ for both comparisons).

\section{Recall}

The mean number of objects recalled from set 1 in the context reminder group was compared to the performance of the context reminder group of Experiment 1, in which recall was tested in the same context. Unexpectedly, subjects in the context group of Experiment $4(M=43.8 \%)$ recalled significantly more objects from set 1 than subjects in the context group of Experiment 1 $\left(M=33.8 \% ; t_{(28)}=2.11, P=0.04\right)$.

\section{Intrusions}

Descriptive analyses of the data indicated that in both groups in Experiment 4, the mean number of intrusions followed a bimodal distribution. Therefore, we performed a median split and analyzed the data in each subgroup separately. Figure 2 displays the mean number of intrusions in each of the subgroups. A comparison between the context reminder subgroups (below median vs. above median) and the context group of Experiment 1 showed that the below-median subgroup showed significantly fewer intrusions than the context group of Experiment $1\left(t_{(13)}=5.39\right.$, $P<0.01$ ), whereas the number of intrusions was similar in the above-median subgroup and the context group of Experiment 1 $(t<1)$.

Similarly, the mean number of intrusions in both threecomponent reminder subgroups (below median vs. above median) were separately compared to the three-component reminder group

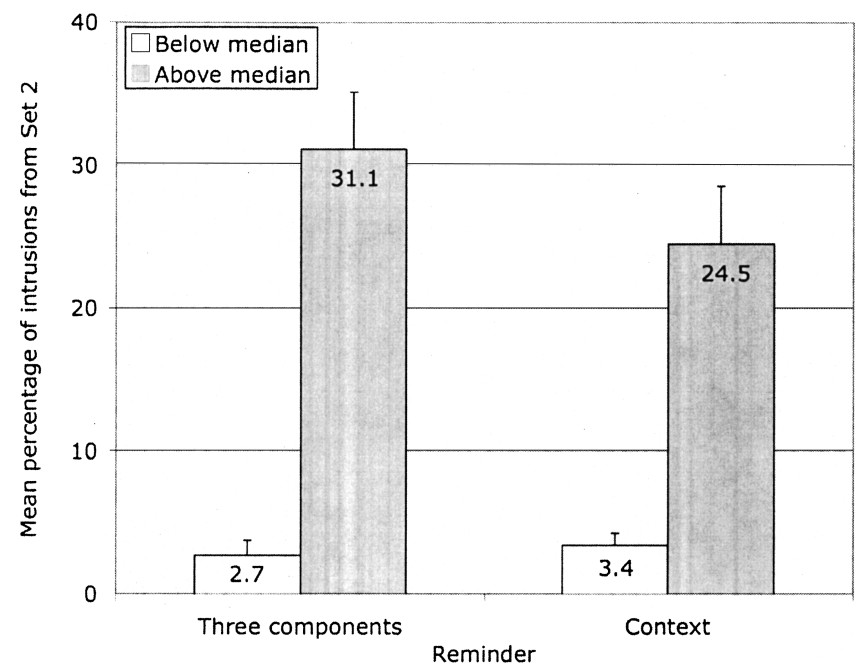

Figure 2. Mean percentage of objects falsely recalled from set 2 in session 3 in Experiment 4 in the two subgroups of each experimental group (three-component reminder vs. context reminder) resulting from a median split performed on the number of intrusions. 
of our previous study, where recall was tested in the same context (Experiment $\left.1, M_{\text {recall }}=36.3 \%, M_{\text {intrusions }}=23.8 \%\right)($ Hupbach et al. 2007): The below median group had significantly fewer intrusions than the corresponding reminder group in our previous study $\left(t_{(12)}=4.94, P<0.01\right)$, whereas the level of intrusions was comparable for the above-median subgroup and the reminder group of our previous study $\left(t_{(16)}=1.27, P=0.22\right)$.

\section{Discussion}

Contrary to previous research on context-dependent memory, recall of set 1 objects was better when it took place in a different context (Experiment 4) than when it took place in the same context (Experiment 1). However, our design is different from that usually employed in the study of context-dependent memory in that subjects in our study learned a second set in the same spatial context in which they had learned the first set. Such interpolated learning is usually not part of the contextdependent memory studies and might have contributed to the reversed effect.

With regard to the updating effect, subjects fell into two groups: Either they showed few intrusions, or they showed a level of intrusions comparable to the updating effect found in our previous study and the context groups of Experiments 1 and 2 of the present study. That at least half of the subjects were able to access the updated memory in a different context shows that updated memories, at least in these subjects, are not context bound. This in turn shows that the critical role of context in triggering the updating effect in Experiments 1-3 reflects characteristics of processes involved in memory encoding and storage rather than the correspondence of training and retrieval conditions.

That a sizable number of subjects did not demonstrate updated memory when tested for recall in a different context is intriguing, and it is at this time unclear what determines whether the updating effect is or is not context bound for a specific individual. As hypothesized above, subjects who show no intrusions might rely on other retrieval cues that are more distinctive for the individual sets of objects. Note that the conditions for our subjects in the first two stages of this study were identical to those for subjects in our earlier studies. Thus, it is reasonable to assume that reactivation triggered some form of memory updating in all our subjects. However, the updated memory was expressed in some subjects, who showed intrusions, and was not expressed in others, who did not show intrusions. Perhaps updating, triggered as it is by the relevant training context (Experiments 1-3), forges a linkage between that context and items from both set 1 and set 2 . When recall takes place in the same context intrusions necessarily result. When recall takes place in another context, intrusions may or may not result, depending on other retrieval cues individual subjects might have generated to help them recall objects in set 1 and set 2 .

\section{General Discussion}

Our studies reveal a unique role of spatial context in the updating of episodic memories. Updating memory of set 1 as reflected by incorporating items from set 2 only occurred when set 2 was learned in the same spatial context as set 1 . Experiment 1 identified the spatial context as a sufficient reminder initiating memory reconsolidation. Furthermore, the failure of the experimenter and the reminder question to induce memory updating was not simply due to these individual components being less powerful reminders; even in combination they did not elicit memory updating. Experiment 2 clearly demonstrates that updating in this paradigm only occurs if the spatial context is part of the reminder compound. Additionally, Experiment 3 shows that neither briefly revisiting the spatial context nor recalling it elsewhere is sufficient for inducing this form of memory mallea- bility. Together, these results indicate that reexposure to the original spatial context is necessary to elicit memory-updating processes. Experiment 4 shows that the modified memory can be recalled outside the original spatial context, although $\sim 50 \%$ of the subjects do not express updated memory in this situation.

We suggest that spatial context is not merely a simple cue but instead plays a superordinate role (Nadel et al. 1985) by providing a scaffold to which experiences are bound. When brought back to the context where the first set of objects was learned, memory for that set is reactivated. This reactivation renders the memory plastic such that it can then be modified and updated readily, by incorporating objects from the second set into the original trace. In contrast, the learning of a second set of objects in a novel context activates an entirely different scaffold, creating a new episodic memory. Thus, subjects who learned set 2 in a different spatial context did not modify the memory for set 1 .

This context-based account of our findings is supported by several empirical observations showing that memory for the context itself is not as malleable as memory for what happens in it. First, spatial context itself does not undergo reconsolidation after reactivation (Biedenkapp and Rudy 2004); instead, it remains unaffected by manipulations that disrupt memory for events experienced in that space. Second, neural representations of context in the hippocampus maintain their stability in the face of minor changes, reflecting the apparent involvement of attractor dynamics (Wills et al. 2005). Major changes, however, lead to complete "remapping" and the creation of an entirely new code for what the organism has decided is a new context.

The critical role of context in determining whether memories of events are reactivated and updated makes sense when one considers that contexts often define events; for instance, the particulars of the current situation may determine which responses are adequate and which are not. However, context-dependent memory change is not always desirable-revisiting the spatial location associated with a witnessed event, for example, might involuntarily lead to memory modifications and distorted eyewitness testimony.

Does spatial context always play an essential role in initiating updating in episodic memory? Or are there boundary conditions on this role? We suspect that familiarity of the spatial context may constitute one such boundary. Familiar contexts are associated with a variety of different experiences; thus, a familiar spatial context may not be particularly useful in distinguishing events. In this case other situational elements, such as the experimenter, may be used to discriminate discrete episodes from one another. Furthermore, it remains to be determined whether scaffolds have to be spatial in nature. For instance, schemas serve as "memory organizers" and have been shown to facilitate memory consolidation (Tse et al. 2007).

Age of memory might constitute another boundary condition. In our study, the memory for the set of objects was only $2 \mathrm{~d}$ old at the time of reactivation. This raises the question of whether the memory was already fully consolidated at the time of reactivation. It could be the case that context-dependent updating only works for memories that are not fully consolidated. Moreover, recent animal studies (Wiltgen and Silva 2007; Winocur et al. 2007) suggest that memories become context independent over time. In these studies, rats were trained in a fear conditioning paradigm in a particular context and tested for retention at various times after learning in either the training context or a new context. As the training-test interval increased, rats increasingly generalized their fear to such novel contexts. These results raise the possibility that the ability of specific contexts to control memory reactivation might diminish over time. Future studies will have to explore the possibility that increased delays between sessions 1 and 2 will allow updating to be triggered in new contexts. 
It is also important to point out that spatial context does not play a critical role for the reconsolidation of other forms of memories, such as auditory fear conditioning in rats that critically depends upon the amygdala. Specifically, auditory fear memories undergo reconsolidation even when animals are exposed to the cue in a novel context (e.g., Nader et al. 2000).

Updating of existing memories, contingent upon reactivation, points to the role of reconsolidation as a general memoryupdating mechanism. Recent findings in rat studies show that post-reminder treatments are effective only when the rat is in an "encoding mode," that is, when new information is presented or expected during the reactivation phase (Morris et al. 2006; Rossato et al. 2007). Furthermore, encoding of new knowledge is different from initial learning (e.g., Lee et al. 2004). Thus, determining how and when the brain shifts in and out of a "novelty encoding" mode is central to understanding how plasticity is induced in memory. The studies presented here take us a step in that direction.

\section{Acknowledgments}

We thank Andrea Gonzalez, Rabiah Muhammad, Auna Otts, Michael Weiss, and Cong Zhang for help with data collection. This research was supported by a NSF CAREER award BCS-0238584 (R.G.) and by the Arizona Alzheimer's Research Consortium and Arizona Department of Health Services, HB2354 (L.N.).

\section{Appendix}

The following objects were presented in session 1: balloon, bow, calculator, toy car, crayon, cup, dice, feather, flashlight, flower, glue, key, sock, sponge, spoon, sunglasses, teabag, tennis ball, toothbrush, and whistle.

The following objects were presented in session 2: apple, band-aid, battery, book, cassette tape, cellular phone, comb, dollar bill, toy elephant, envelope, paper clip, toy pot, puzzle piece, rock, straw, thread, tissue, watch, toy shovel, and zipper.

\section{References}

Biedenkapp, J.C. and Rudy, J.W. 2004. Context memories and reactivation: Constraints on the reconsolidation hypothesis. Behav. Neurosci. 118: 956-964.

Debiec, J., Doyere, V., Nader, K., and LeDoux, J.E. 2006. Directly reactivated, but not indirectly reactivated, memories undergo reconsolidation in the amygdala. Proc. Natl. Acad. Sci. 103: $3428-3433$

Dudai, Y. 2006. Reconsolidation: The advantage of being refocused. Curr. Opin. Neurobiol. 16: 174-178.
Hassabis, D., Kumaran, D., Vann, S.D., and Maguire, E.A. 2007. Patients with hippocampal amnesia cannot imagine new experiences. Proc. Natl. Acad. Sci. 104: 1726-1731.

Hupbach, A., Gomez, R., Hardt, O., and Nadel, L. 2007. Reconsolidation of episodic memories: A subtle reminder triggers integration of new information. Learn. Mem. 14: 47-53.

Lee, J.L.C., Everitt, B.J., and Thomas, K.L. 2004. Independent cellular processes for hippocampal memory consolidation and reconsolidation. Science 304: 839-843.

McGaugh, J.L. 2000. Memory-A century of consolidation. Science 287: $248-251$.

Misanin, J.R., Miller, R.R., and Lewis, D.J. 1968. Retrograde amnesia produced by electroconvulsive shock after reactivation of a consolidated memory trace. Science 160: 554-555.

Morris, C.D., Bransford, J.D., and Franks, J.J. 1977. Levels of processing versus transfer appropriate processing. J. Verbal Learn. Verbal Behav. 16: $519-533$.

Morris, R.G.M., Inglis, J., Ainge, J.A., Olverman, H.J., Tulloch, J., Dudai, Y., and Kelly, P.A. 2006. Memory reconsolidation: Sensitivity of spatial memory to inhibition of protein synthesis in dorsal hippocampus during encoding and retrieval. Neuron 50: 479-489.

Nadel, L. and Willner, J. 1980. Context and conditioning: A place for space. Physiol. Psychol. 8: 218-228.

Nadel, L., Willner, J., and Kurz, E.M. 1985. Cognitive maps and environmental context. In Context and learning (eds. P. Balsam and A. Tomie), pp. 385-406. LEA, Hillsdale, NJ.

Nader, K., Schafe, G.E., and Le Doux, J.F. 2000. Fear memories require protein synthesis in the amygdala for reconsolidation after retrieval. Nature 406: 722-726.

O'Keefe, J. and Nadel, L. 1978. The hippocampus as a cognitive map. Oxford University Press, Oxford.

Rossato, J.I., Bevilagua, L.R., Myskiw, J.C., Medina, J.H., Izquierdo, I. and Cammarota, M. 2007. On the role of hippocampal protein synthesis in the consolidation and reconsolidation of object recognition memory. Learn. Mem. 14: 36-46.

Smith, S.M. and Vela, E. 2001. Environmental context-dependent memory: A review and meta-analysis. Psychon. Bull. Rev. 8: 203-220.

Tse, D., Langston, R.F., Kakeyama, M., Bethus, I., Spooner, P.A., Wood, E.R., Witter, M.P., and Morris, R.G. 2007. Schemas and memory reconsolidation. Science 316: 76-82.

Tulving, E. and Thomson, D.M. 1973. Encoding specificity and retrieval processes in episodic memory. Psychol. Rev. 80: 352-373.

Walker, M.P., Brakefield, T., Hobson, J.A., and Stickgold, R. 2003. Dissociable stages of human memory consolidation and reconsolidation. Nature 425: 616-620.

Wills, T.J., Lever, C., Cacucci, F., Burgess, N., and O'Keefe, J. 2005. Attractor dynamics in the hippocampal representation of the local environment. Science 308: 873-876.

Wiltgen, B.J. and Silva, A.J. 2007. Memory for context becomes less specific with time. Learn. Mem. 14: 313-317.

Winocur, G., Moscovitch, M., and Sekeres, M. 2007. Memory consolidation or transformation: Context manipulation and hippocampal representations of memory. Nat. Neurosci. 10: 555-557.

Received April 8, 2008; accepted in revised form June 1, 2008. 


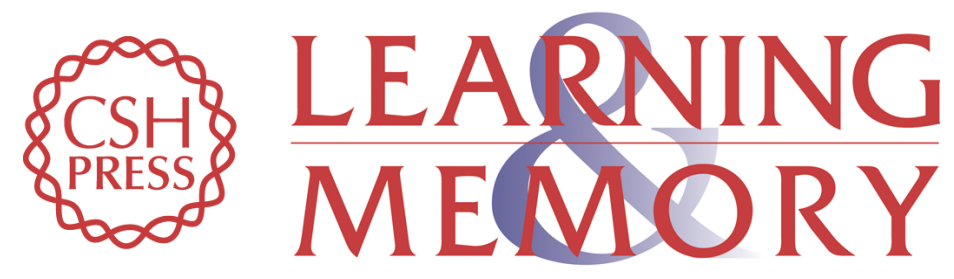

\section{The dynamics of memory: Context-dependent updating}

Almut Hupbach, Oliver Hardt, Rebecca Gomez, et al.

Learn. Mem. 2008, 15:

Access the most recent version at doi:10.1101//m.1022308

References This article cites 20 articles, 10 of which can be accessed free at: http://learnmem.cshlp.org/content/15/8/574.full.html\#ref-list-1

License

Email Alerting Receive free email alerts when new articles cite this article - sign up in the box at the Service top right corner of the article or click here. 